\title{
DIAGNOSTIC POTENTIAL OF WESTERN BLOT ANALYSIS OF SERA FROM DOGS WITH LEISHMANIASIS IN ENDEMIC AREAS AND SIGNIFICANCE OF THE PATTERN
}

\author{
M. JESUS AISA, SOLEDAD CASTILLEJO, MONTSERRAT GALLEGO, ROSER FISA, M. CRISTINA RIERA, \\ MARIA DE COLMENARES, SARA TORRAS, XAVIER ROURA, JOAN SENTIS AND MONTSERRAT PORTUS \\ Laboratori de Parasitologia, Departament de Microbiologia i Parasitologia Sanitaries, i Unitat de Bioestadistica, \\ Departament de Salut Publica, Universitat de Barcelona, Barcelona, Spain; Hospital Clinic de Veterinaria, \\ Facultat de Veterinaria, Universitat Autonoma de Barcelona, Barcelona, Spain
}

\begin{abstract}
Serum samples collected from 237 dogs in Catalonia (northeastern Spain) were screened by Western blot analysis to detect the presence of antibodies specific to different Leishmania infantum polypeptide fractions. Leishmaniasis was confirmed in 72 of these dogs by direct examination and/or culture. Another 165 animals from the Priorat region were studied periodically for 2-8 years between 1987 and 1995, giving a total of 565 determinations. A control group of $93 \mathrm{dogs}$ from nonendemic areas was also studied. Sera from dogs with leishmaniasis recognized antigens with molecular weights ranging from 12 to $85 \mathrm{kD}$. The most sensitive antigens were those of 70, 65, 46, $30,28,14$, and $12 \mathrm{kD}$, which were recognized by $75 \%, 75 \%, 78 \%, 75 \%, 81 \%, 79 \%$, and $75 \%$, respectively, of the sera from dogs with positive parasitologic examination results. Antigens of 70 and $65 \mathrm{kD}$ were also recognized by two dogs from nonendemic areas. Antigens of 14 and $12 \mathrm{kD}$ were the first to be recognized by sera of asymptomatic dogs with titers less than the cut-off value of the dot-ELISA that increased during the longitudinal study, and the presence of antibodies specific for these fractions was observed for up to six years before seroconversion observed by dot-ELISA. These antibodies were also the first to disappear in dogs in which the disease was self-limited. The study corroborates the high sensitivity and specificity of Western blots in the diagnosis of canine leishmaniasis when the bands of low molecular weight (less than $46 \mathrm{kD}$ ) are considered, and indicates that fractions of 14 and $12 \mathrm{kD}$ are useful in detecting early forms of the disease.
\end{abstract}

Canine leishmaniasis is endemic in Spain, where approximately $5 \%$ of the dog population are infected. Diagnosis of the disease relies on direct examination and culture of lymph nodes or bone marrow aspirates and serologic techniques.

Interpretation of results obtained by serologic techniques (ELISA, dot-ELISA, immunofluorescence assay [IFA]) when dogs from endemic areas are examined is often difficult. Although these techniques can detect active leishmaniasis when a high level of specific antibodies is present, they do not distinguish between the various phases of the disease when levels of antibodies are at or near the cut-off level. Different seroepidemiologic studies performed in the Mediterranean area $^{1-4}$ indicate that the proportion of sera with low antibody titers is directly related to the level of endemicity. The study of the natural evolution of leishmaniasis in the Priorat focus of northeastern Spain by a dot-ELISA technique showed that $40 \%$ of the dogs examined had low antibody titers. ${ }^{5}$ This low level of antibodies may be a consequence of the latent period of the disease, which can last from a few months to several years, abortive or regressive forms of the disease, a low humoral immunoresponse in the animal, or different host-parasite contacts without the establishment of the parasite. Distinguishing between these situations is of great importance clinically and epidemiologically.

Recent studies ${ }^{6-8}$ reported different results concerning the pattern obtained in Western blot analysis of the specific humoral immunoresponse to $L$. infantum antigens during the active course of the disease and during therapy in human leishmaniasis. Limited studies have been performed on dogs, ${ }^{7,9-12}$ and little is known about the natural evolution of this immunoresponse during active or latent asymptomatic canine infection, or during regression of the disease.

The aim of this study was to define the polypeptide fractions of the Leishmania antigen that might be used in the diagnosis of the disease, and to determine whether the pat- tern obtained in Western blots could be used as a marker for the evolution of disease.

\section{MATERIALS AND METHODS}

Sera. Sera were obtained from $237 \mathrm{dogs}$ in Catalonia (northeastern Spain), an area endemic for leishmaniasis, 72 of which had leishmaniasis as confirmed by direct examination and/or culture. Forty-one were treated at the Veterinary Hospital of the Facultat de Veterinaria de Bellaterra in Barcelona, and 31 were from a focus of leishmaniasis in Priorat.

Sera from 165 animals from the natural focus in Priorat (south of Catalonia) were periodically studied for 2-8 years between 1987 and 1995, giving a total of 565 determinations, with a mean of 3.4 determinations per dog (range $=$ 2-8). Clinical examination and blood collection were carried out during the annual antirabies vaccination campaign. Complete records of breed, sex, age, and size, were obtained for epidemiologic purposes and clinical signs for leishmaniasis were evaluated. Dogs were classified as asymptomatic when there was no indication of Leishmania infection (moderately enlarged lymph nodes found in a few cases were disregarded); oligosymptomatic when moderate symptoms, including two signs of infection (enlarged lymph nodes and onychogryposis or furfuraceous dermatitis/depilation) were observed; and symptomatic when evident symptoms including three or more signs of disease were observed.

Animals were examined at annual intervals. Many of the animals died during the study without the cause of death being established, and a large number of animals were also lost during the study for a variety of reasons, such as changes in residence and lack of permission of the owner to continue the study. Parasitologic examination (direct examination and culture) could be performed only in some cases due to difficulties in collecting repeated ganglionic aspirates 
under field conditions and from apparently healthy dogs. A control group of $93 \mathrm{dogs}$ from nonendemic areas (Switzerland, Sweden, and the Canary Islands) was also studied.

Blood samples were allowed to clot, and the serum was separated by centrifugation and stored at $-40^{\circ} \mathrm{C}$ until use.

Dot-ELISA. The technique was performed as previously described. ${ }^{13}$ Briefly, the antigen used was whole promastigotes of $L$. infantum fixed in $1.5 \%$ formaldehyde. Dots of 1 $\mu \mathrm{l}$ containing $10^{5}$ cells were adsorbed onto a nitrocellulose membrane in a bio-dot apparatus (Bio-Dot ${ }^{\mathrm{WW}}$; Bio-Rad, Hercules, CA). Fifty microliters of serially diluted serum samples (from 1:100) in $20 \mathrm{mM}$ Tris, $0.13 \mathrm{mM} \mathrm{NaCl}, \mathrm{pH} \mathrm{7.6}$, containing $0.05 \%$ Tween 20 (TST) and $1 \%$ dry skim milk was added to each well and incubated at $37^{\circ} \mathrm{C}$ for $30 \mathrm{~min}$. After the dots were washed with TST, $50 \mu$ l of Protein A labeled with horseradish peroxidase (Sigma, St. Louis, MO) diluted 1:1,000 in TST was added and incubated at $37^{\circ} \mathrm{C}$ for $30 \mathrm{~min}$. After washing with TST, the entire membrane was exposed to the chromogen reagent (4-chloro-1-naphthol [Sigma]) and $\mathrm{H}_{2} \mathrm{O}_{2}$. Color development was visually determined and the cut-off value was established at a 1:800 dilution of serum.

Immunoblot analysis. Antigen for immunoblot was obtained from promastigotes from culture in Schneider's medium (Sigma) containing 20\% fetal calf serum at the exponential growth phase. Cells were washed three times in phosphate-buffered saline, $\mathrm{pH} 7.4$, counted, and adjusted to a concentration of $3 \times 10^{8}$ promastigotes $/ \mathrm{ml}$ in sample buffer (0.5 M Tris-HCl, pH 6.8, 0.01 M EDTA, 5\% sodium dodecyl sulfate [SDS], 5\% 2-mercaptoethanol, 0.0125\% bromophenol blue), and boiled for $5 \mathrm{~min}$. Antigen was stored at $-40^{\circ} \mathrm{C}$ until use.

Antigen electrophoresis on $0.1 \%$ SDS- $15 \%$ polyacrylamide gels together with molecular mass proteins standards (Standard Low Range; Bio-Rad) was performed on a MiniGel AE 6400 Dual Mini Slab Kit (Atto, Bunkyo-ku, Japan). Gels were run at $80 \mathrm{~V}$ for $2 \mathrm{hr}$ at room temperature. Polypeptides were transblotted onto nitrocellulose sheets $(0.45-$ $\mu \mathrm{m}$ pore size, HAWP 304 FO; Millipore, Bedford, MA) and the sheets were blocked with $20 \mathrm{mM}$ Tris, $0.13 \mathrm{mM} \mathrm{NaCl}$, $\mathrm{pH} 7.6$ (TS), 5\% skim milk overnight at $4^{\circ} \mathrm{C}$. The sheets were washed in TS and introduced into a multiscreen apparatus (Mini Protean II, Multiscreen Apparatus; Bio-Rad). Sera were diluted 1:200 in TS-1\% skim milk, $0.2 \%$ Tween 20 , and $500 \mu l$ of each sample was introduced into each channel of the multiscreen apparatus and incubated for $2 \mathrm{hr}$ at $37^{\circ} \mathrm{C}$. Bound immunoglobulins were developed by incubation with a 1:1,000 dilution of Protein A peroxidase conjugate (Sigma) for $1 \mathrm{hr}$. After the sheets were washed three times with TST and a final time with TS, color was developed with 4-chloro-1-naphthol (Sigma) and $\mathrm{H}_{2} \mathrm{O}_{2}$ and the reaction was stopped with tap water after $30 \mathrm{~min}$.

Statistical analysis. The relationship between the different antigen fractions recognized on Western blots and the presence of the parasite in dogs was determined by the chisquare test. The intensity of this relationship was calculated with Pearson's coefficient of contingency. Any $P$ values $<$ 0.05 were considered statistically significant.

\section{RESULTS}

Seventy of 72 dogs with leishmaniasis had antibodies that revealed 3-33 polypeptide fractions of the Leishmania an-
TABLE 1

Recognition of the major Leishmania infantum antigens by sera of 72 dogs with leishmaniasis, sensitivity for each band in the diagnosis, its relationship with the presence of parasite $\left(\chi^{2}\right)$, and intensity of this relation (C-Pearson's coefficient of contingency)

\begin{tabular}{ccrc}
\hline Band $(\mathrm{kD})$ & Sensitivity $(\%)$ & \multicolumn{1}{c}{$\chi^{2}$} & $\mathrm{C}$ \\
\hline 85 & 13.90 & 14.16 & 0.279 \\
80 & 52.78 & 66.61 & 0.530 \\
75 & 65.28 & 80.90 & 0.567 \\
70 & 75.00 & 103.79 & 0.615 \\
68 & 26.39 & 29.11 & 0.382 \\
65 & 75.00 & 103.79 & 0.615 \\
63 & 36.11 & 41.77 & 0.444 \\
60 & 65.28 & 80.90 & 0.567 \\
58 & 30.55 & 30.94 & 0.392 \\
56 & 45.83 & 55.73 & 0.496 \\
54 & 25.00 & 27.40 & 0.372 \\
52 & 29.17 & 32.60 & 0.401 \\
50 & 18.05 & 19.15 & 0.318 \\
48 & 41.67 & 49.58 & 0.475 \\
46 & 77.78 & 113.66 & 0.633 \\
44 & 51.39 & 64.37 & 0.524 \\
42 & 68.05 & 93.70 & 0.596 \\
40 & 65.28 & 88.41 & 0.584 \\
38 & 61.11 & 80.80 & 0.567 \\
36 & 65.28 & 88.41 & 0.584 \\
34 & 52.78 & 62.89 & 0.519 \\
32 & 65.28 & 88.41 & 0.584 \\
30 & 75.00 & 107.71 & 0.622 \\
28 & 80.55 & 88.41 & 0.642 \\
26 & 58.33 & 47.59 & 0.555 \\
24 & 37.50 & 43.68 & 0.452 \\
22 & 40.27 & 75.92 & 0.467 \\
20 & 37.50 & 43.68 & 0.452 \\
18 & 31.94 & 36.20 & 0.418 \\
16 & 31.94 & 36.20 & 0.418 \\
14 & 79.17 & 116.71 & 0.635 \\
12 & 75.00 & 107.71 & 0.626 \\
\hline
\end{tabular}

tigen with a molecular mass range between 12 and $85 \mathrm{kD}$. The highest sensitivity was found for bands of 70, 65, 46, $30,28,14$, and $12 \mathrm{kD}$ (Table 1).

The specificity of the technique was calculated with the sera of 93 dogs from nonendemic areas. Sera of four dogs from Sweden reacted with some fractions of the Leishmania antigen (75-70, 75-60, 65-60, 58, and $34 \mathrm{kD}$, respectively). The coefficient of contingency for each band and the presence of the parasite was calculated (Table 1), and the strongest relationship was found for bands of 46, 30, 28, 14, and $12 \mathrm{kD}$. Based on these results, we considered the Western blot to be positive when any one of the latter bands was present and the sensitivity of the technique was calculated to be $95.8 \%$.

None of the animals negative by Western blot was positive by dot-ELISA $(>1: 400)$ and although a direct relationship between the results obtained in the dot-ELISA and the Western blot could not be established, in general, the number of bands in the immunoblot increased with the titer obtained by the dot-ELISA (Figure 1). The sensitivity obtained with this technique for the same animals was $91.6 \%$.

Epidemiologic study. One hundred sixty-five dogs from the Priorat focus of leishmaniasis were studied serologically (Western blot and dot-ELISA) for 2-8 years. With regards to the serologic evolution of the animals, we observed the following results. 1) Seventy-four of 165 dogs (group A) 


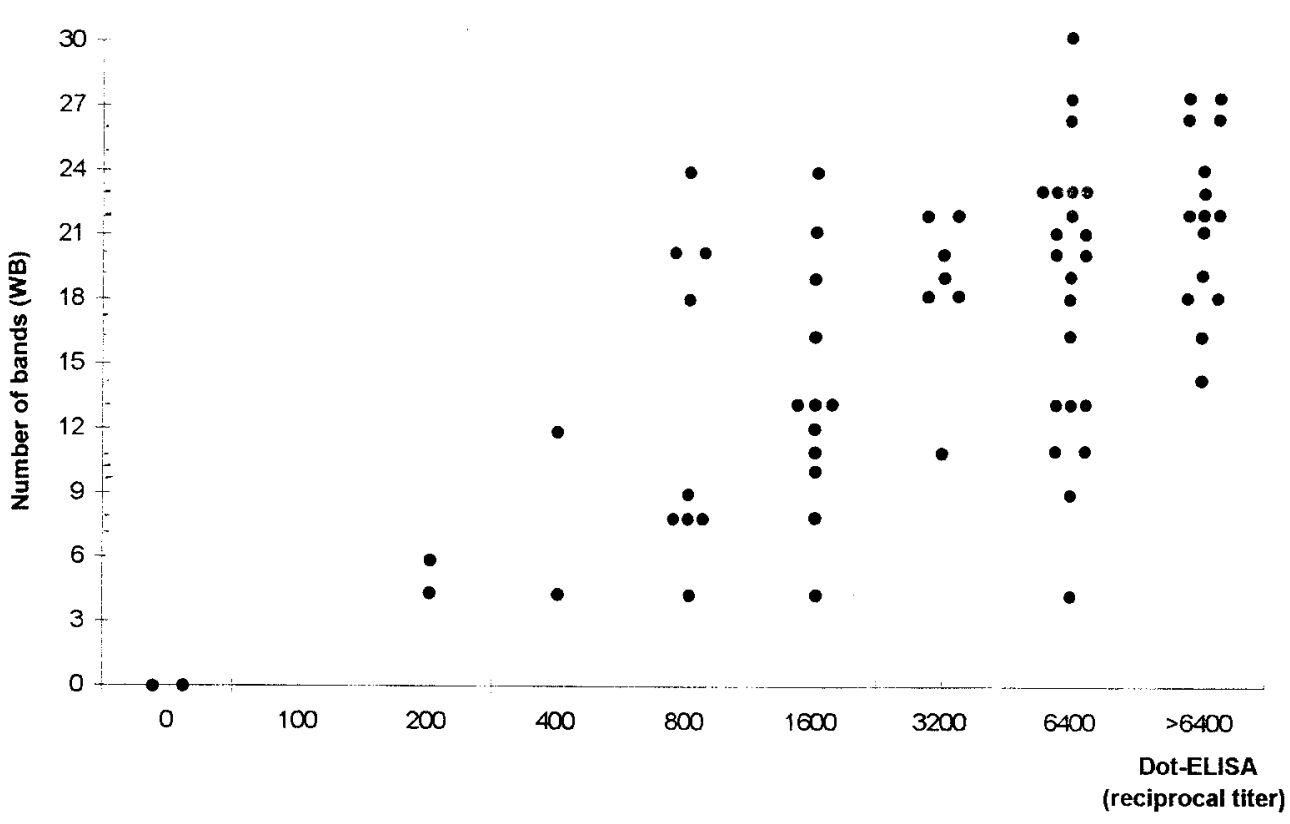

FIGURE 1. Comparison of the results obtained by dot-ELISA and Western blot (WB) in 72 dogs with leishmaniasis confirmed by direct examination and/or culture.

were negative throughout the study by Western blot (sera did not reveal any antigen band of Leishmania) and negative by dot-ELISA or less than the cut-off value. This group can be considered as the cohort of negative animals and none of them had symptoms of Leishmania infection. 2) Thirteen of 165 dogs (group B) remained Western blot positive throughout the study. The symptoms of these dogs changed during
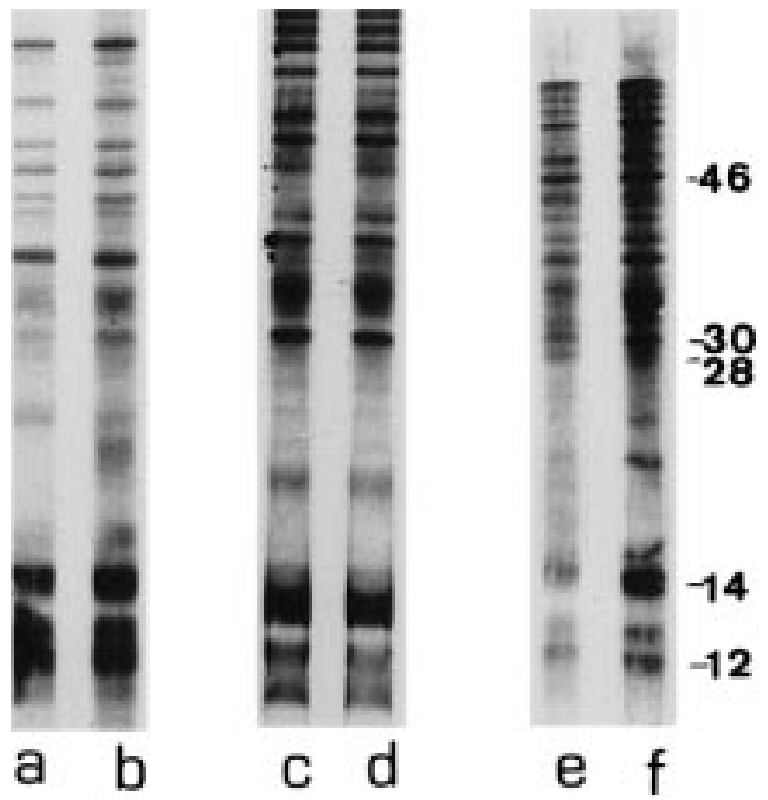

FIGURE 2. Western blot analysis of sera from three positive dogs (dot-ELISA, Western blot, and culture). Lanes a and b, dog no. 36; a, 1991, dot-ELISA 1:1,600, asymptomatic; b, 1992, dot-ELISA 1: 1,600 , asymptomatic. Lanes $\mathrm{c}$ and d, dog no. 31; c, 1991, dot-ELISA 1:6,400, asymptomatic; d, 1992, dot-ELISA 1:6,400, symptomatic. Lanes e and f, dog no. 18; e, 1991, dot-ELISA 1:3,200, asymptomatic; f, 1992, dot-ELISA 1:3,200, asymptomatic. All dogs died in 1993. Values on the right are in kilodaltons. the study; eight were asymptomatic at the beginning of the study but only three of them remained healthy at the last examination (Figure 2). 3) Twenty-nine of 165 dogs (group C) seroconverted. In these cases, the number of antigenic fractions recognized increased gradually after the first appearance of bands of 12 and $14 \mathrm{kD}$ (e.g., dogs 58, 140, and 145, Figure 3). Nineteen of 29 were asymptomatic at all times, whereas 10 of 29 were oligosymptomatic at the last examination. 4) Seven of 165 dogs (group D) showed a clear reduction in the number of bands, together with a decrease in the dot-ELISA titer. These animals were always asymptomatic or oligosymptomatic; this was accompanied by a reduction and/or disappearance of bands of low molecular weights, (e.g., dogs 113 and 140, Figure 3). In two cases there was an increase and a later reduction in the number of bands in Western blots and in the titer of the dot-ELISA (these animals are included in both groups $\mathrm{C}$ and $\mathrm{D}$ ). We believe that this corresponds to the self-healing population. 5) Serum observed from 38 of 165 dogs (group E) showed at certain times proteins corresponding to bands not included among those considered diagnostic $(46,30,28,14$, and 12 $\mathrm{kD})$. All of these dogs were negative by dot-ELISA or had an antibody level less than the cut-off value $(<1: 800)$, and none became positive (either by dot-ELISA or Western blot) during the period of the study (Figure 4). Twelve of the 38 dogs showed a single band of $70 \mathrm{kD}$. This single band was also seen in the serum of one dog in group D (number 113, Figure 3), which later became seronegative (by dot-ELISA and Western blot) for three consecutive years. 6) Six of 165 dogs (group F) were observed that could not be included in any of the above groups.

The relationship between results in the dot-ELISA and Western blots for each sera and the clinical status of the animals at the time of sampling is shown in Table 2. Most sera with a negative dot-ELISA result (375) also had a negative Western blot result (365), and most sera with a positive 


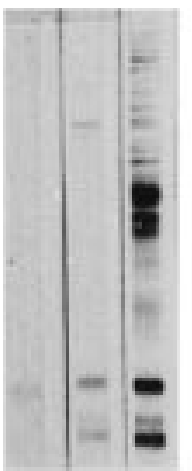

a b c
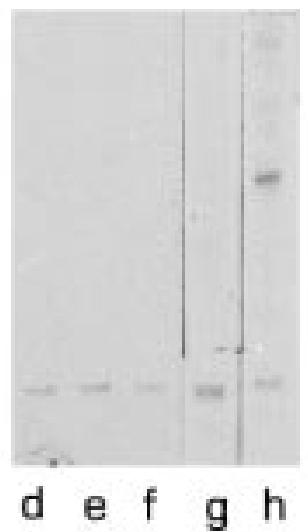

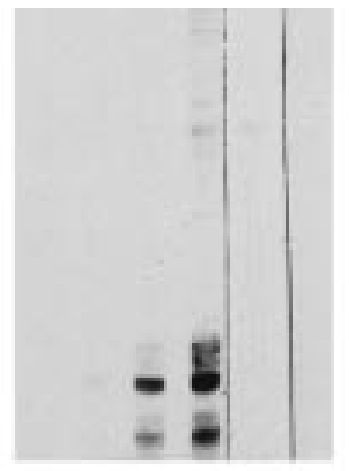

i j $k$ I m n

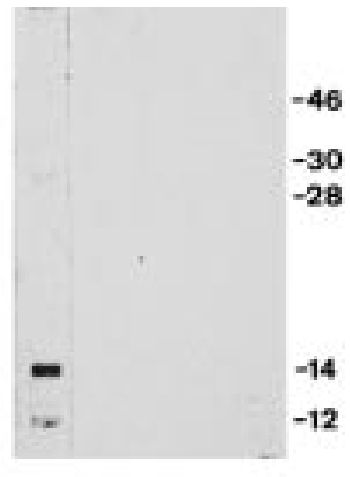

o $\quad p \quad q \quad r \quad s$

FIGURE 3. Western blot analysis of serum of four dogs in which the titer in the dot-ELISA and the Western blot pattern changed over time. Lanes a-c, dog no. 58; a, 1990, dot-ELISA 1:100; b, 1991, dot-ELISA 1:1,600; c, 1992, dot-ELISA 1:6,400; the dog remained asymptomatic Lanes d-h, dog no. 145; d, 1988, dot-ELISA 1:200; e, 1989, dot-ELISA 1:400; f, 1991, dot-ELISA 1:800; g, 1992, dot-ELISA 1:800; h, 1993, dot-ELISA 1:800; the dog remained asymptomatic with the exception of 1992 when slight adenopatia and scattered depilations on the skin were observed. Lanes i-n, dog no. 140; i, 1989, dot-ELISA negative; j, 1990, dot-ELISA 1:100; k, 1991, dot-ELISA 1:200; 1, 1992, dotELISA 1:800; m, 1993, dot-ELISA 1:100; $\mathrm{n}, 1994$, dot-ELISA 1:100; cultures of lymph node aspirates and bone marrow were positive in 1992 and the dog remained asymptomatic in the control period. Lanes o-s, dog no. 113; o, 1991, dot-ELISA 1:400; p, 1992, dot-ELISA 1: 100; q, 1993, dot-ELISA negative; r, 1994, dot-ELISA negative; s, 1995, dot-ELISA negative; the dog remained asymptomatic with the exception of the control in 1995, which had depilations on the ears. Values on the right are in kilodaltons.

dot-ELISA result (61) also had a positive Western blot result (57). Only four sera positive by dot-ELISA had a negative Western blot result. In all cases these dot-ELISA titers were near the cut-off value $(1: 800)$ and Western blots revealed bands other than those considered diagnostic. One of these dogs became positive by Western blot and the dot-ELISA titer increased to 1:3,200 in a second control; another dog died the following year of undetermined causes and two retained dot-ELISA titers less than the cut-off value and Western blots with nonspecific bands for next two and three years, respectively. Ten sera from eight asymptomatic dogs with negative dot-ELISA results had positive Western blots; five dogs were from group $\mathrm{C}$ and the bands were 14 or 12 $14 \mathrm{kD}$, and seroconversion by dot-ELISA was observed after a variable period of time. For the remaining three dogs, no additional data was available to evaluate the serology.

One-hundred twenty-nine sera with a dot-ELISA titer less than the cut-off value corresponded to dogs included in all the categories established above. In 58 sera, the Western blot was negative and corresponded to dogs in group A. Another 37 sera were positive by Western blot and recognized a variable pattern of antigenic fractions; 14 of them showed reac-
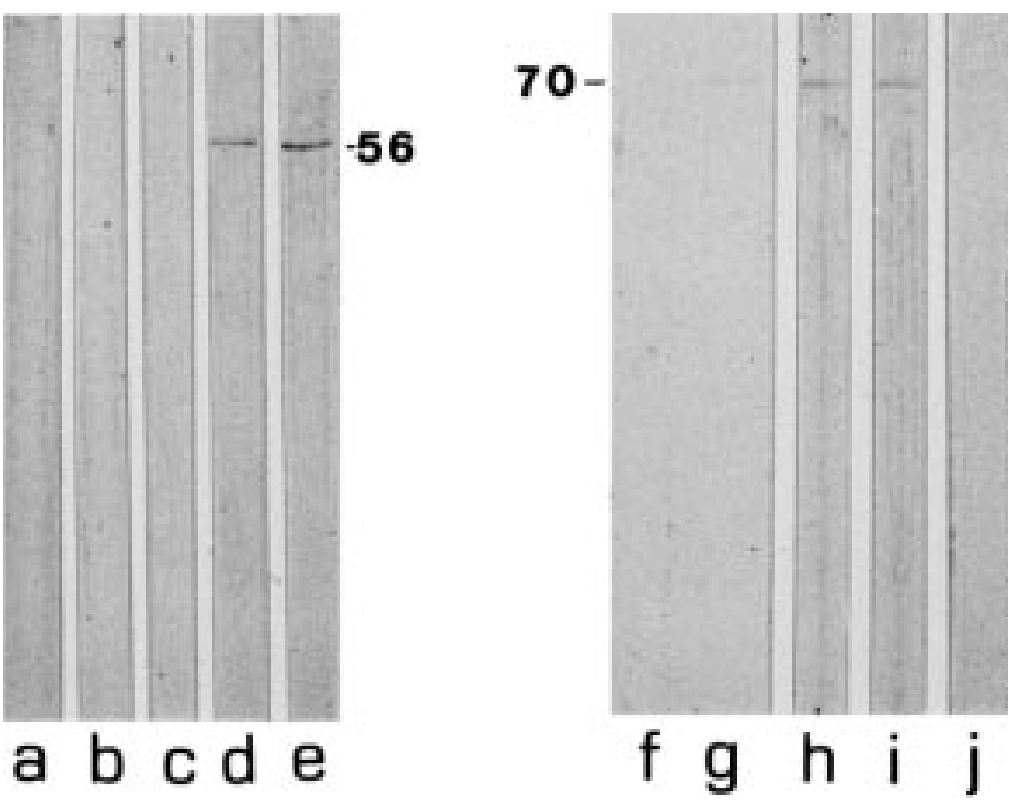

FigURE 4. Western blot analysis of two asymptomatic dogs with dot-ELISA titers that were negative or less than the cut-off value and did not seroconvert. Lanes a-e, dog no. 94; a, 1989, dot-ELISA 1:100; b, 1991, dot-ELISA 1:200; c, 1992, dot-ELISA 1:100; d, 1993, dot-ELISA negative; e, 1994, dot-ELISA 1:100. Lanes f-j, dog no. 59: years 1990, 1991, 1992, and 1994; dot-ELISA was negative in all controls. Values are in kilodaltons. 
TABLE 2

Comparison of the results obtained in the Western blot (WB) and dot-ELISA in sera of asymptomatic, oligosymptomatic, and asymptomatic dogs from the Priorat region of Spain*

\begin{tabular}{|c|c|c|c|c|c|c|}
\hline \multirow[b]{2}{*}{ Dot-ELISA } & \multicolumn{2}{|c|}{$\begin{array}{l}\text { Asymptomatic } \\
(\mathrm{n}=510)\end{array}$} & \multicolumn{2}{|c|}{$\begin{array}{c}\text { Oligosymptomat- } \\
\text { ic } \\
((n)=42)\end{array}$} & \multicolumn{2}{|c|}{$\begin{array}{l}\text { Symptomatic } \\
\quad(\mathrm{n}=13)\end{array}$} \\
\hline & WB- & $\mathrm{WB}+$ & WB- & $\mathrm{WB}+$ & WB- & $\mathrm{WB}+$ \\
\hline Negative $(\mathrm{n}=375)$ & 350 & 10 & 15 & 0 & 0 & 0 \\
\hline $1: 100-1: 400(\mathrm{n}=129)$ & 82 & 31 & 10 & 4 & 0 & 2 \\
\hline$>1: 400(\mathrm{n}=61)$ & 3 & 34 & 1 & 12 & 0 & 11 \\
\hline
\end{tabular}

* The dot-ELISA was considered positive when the titer was $>1: 400$. The Western blot was considered positive when any one of the fractions of $12,14,28,30$, and $46 \mathrm{kD}$ of Leishmania infantum antigen was revealed.

tivity only to the 14 or $12-14 \mathrm{kD}$ bands in dogs in group C. Low titers in the dot-ELISA and positivity by Western blot were also observed in sera obtained from dogs in group D. The remaining 34 sera with low dot-ELISA titers recognized bands not considered diagnostic in dogs in group $\mathrm{E}$, and exceptionally, in group D.

Dogs with symptoms of Leishmania infections were always positive by Western blot and the pattern of antigenic fractions recognized was not different from that recognized by sera obtained during the asymptomatic phases of the disease when antibody levels were similar (Figure 2).

\section{DISCUSSION}

Immunoblot analysis has been recently found to be suitable for the diagnosis of visceral leishmaniasis in humans and in dogs. However, some investigators disagree as to the most specific and sensitive antigen bands recognized by sera of patients and animals with leishmaniasis. ${ }^{6-12}$ Differences in the antigens used, promastigotes from the exponential or stationary growth phase, different degrees of antigen reduction as a consequence of different concentrations of SDS and 2mercaptoethanol in the sample buffer, and separation of the soluble fractions of the antigen and the pellet are some of the factors that could influence the results. Moreover, the size of the slab gels and the concentration of polyacrylamide used, as well as different molecular weight markers, may introduce slight variations in the separation of the fractions and in the relative molecular weights calculated.

The results obtained here are consistent with those obtained by Mary and others ${ }^{6}$ in patients with visceral leishmaniasis in which antigens of 14, 16, 28, 30, 46, and $68 \mathrm{kD}$ were recognized by $92 \%, 95 \%, 63 \%, 80 \%, 69 \%$, and $89 \%$ of the patients' sera, and those of Mancianti and others ${ }^{11}$ in dogs, who found that all positive sera, even when the IFA titer was relatively low, recognized antigens of 30 and 73 $\mathrm{kD}$. Other antigens frequently recognized were those with molecular weights of $16,18,26,33,50$, and $117 \mathrm{kD} .{ }^{11}$ There may be identity between the 12-, 14-, 28-, 30-, 46-, and 70$\mathrm{kD}$ antigens in the present study and the 14-, 16-, 28-, 30-, 46-, and $68-\mathrm{kD}$ antigens reported by Mary and others ${ }^{6}$ and the 16-, 18-, 30-, 33-, 50-, and 73-kD antigens of Mancianti and others. ${ }^{11}$ In a recent study, proteins of 14 and $16 \mathrm{kD}$ have been identified as nuclear proteins and designated p14 and $\mathrm{p} 18 .{ }^{14}$

The calculation of the sensitivity of the test in this study was performed on dogs with positive parasitologic exami- nation results and with symptoms compatible with leishmaniasis. This combination of clinical signs and positive parasitologic examination results usually arises in advanced active diseases. This is why in most of these dogs, the dotELISA results were positive and the Western blots revealed a large number of bands (Figure 1). The epidemiologic study was performed on the canine population of the Priorat area that participated in an antirabies vaccination campaign and were not necessarily ill. In this case, many dogs were in the early subclinical phases of the disease. In rural areas of Catalonia such as the area studied, few dogs are specifically treated for leishmaniasis and many are killed when they are in the advanced phases of the disease. The study of serum in early phases, using techniques that do not distinguish between antigenic fractions (IFA, ELISA, dot-ELISA, direct agglutination test) often results in low titers that cannot define disease or a healthy condition.

Based on the results of this study, it appears that the Western blot is able to discriminate early phases of the disease in animals that are negative or have low antibody titers with the techniques mentioned above. The presence of antibodies specific to the 12 - and $14-\mathrm{kD}$ fractions in asymptomatic dogs may last for a variable period of time and positivity by dotELISA and/or clinical disease has been observed up to six years after detecting the presence of these specific antibodies. This is in agreement with previous observations that when healthy dogs seroconverted, the first bands to appear on Western blots were those of 14 and $16 \mathrm{kD} .^{6}$ Moreover, the long period during which such bands may be found in asymptomatic dogs before they reach the cut-off level of the dot-ELISA could explain previous findings that fractions of low molecular weight were recognized by human controls in endemic areas in France ${ }^{6}$ and Spain, ${ }^{8}$ but not by humans from nonendemic regions.

Dogs that showed a regression of the disease and of antibody titers (group D) had a reduced activity to certain antigen bands: those to the 12- and 14-kD bands disappeared or were reduced in intensity while others to higher molecular weight bands remained. This suggests that in some cases the presence of specific antibodies to antigens of high molecular weight $(>46 \mathrm{kD})$ could be related to previous contact with the parasite. Nevertheless, the significance of some bands of high molecular weights, in particular those of 60, 65, 70, and $75 \mathrm{kD}$, which often appear associated, is not clear because they were sporadically recognized by sera of dogs from nonendemic areas. These bands were also recognized by a high proportion of human negative control sera in Catalonia $^{8}$ and southern France, ${ }^{6}$ which are areas endemic for leishmaniasis. Proteins of 70- and 72-kD in Leishmania antigens have been identified as members of the highly conserved heat-shock protein 70 (hsp70) family, and they are recognized not only by most human visceral leishmaniasis sera but also by sera from patients with other infectious diseases, such as tuberculosis, toxoplasmosis, and hydatidosis. ${ }^{15}$ The response in dogs to hsp70 during leishmaniasis is specifically elicited by the parasite protein and is not an autoimmune reaction. ${ }^{16}$ Nevertheless, the similarity between hsp70 from different infectious agents could explain the presence of cross-reactive antibodies.

This study corroborates the high sensitivity $(95.8 \%)$ and specificity $(100 \%)$ of Western blots for the diagnosis of ca- 
nine leishmaniasis when antigen bands with molecular weights of $12,14,28,30$, and $46 \mathrm{kD}$ are considered. The main advantage of Western blots over other serologic techniques that use whole Leishmania antigen is in its capacity to discriminate early asymptomatic infections. Identification of the 12- and 14-kD antigen fractions of Leishmania in dogs can be used to detect early phases of this disease, which can last many years, and possibly to evaluate the resolution of the disease.

Acknowledgments: We acknowledge the facilities provided by veterinarians, local councils, and people from the Priorat region. We also thank Professors A. Uggla (Uppsala, Sweden), C. Bordier (Laussane, Switzerland), and B. Valladares (La Laguna, Canary Islands) for providing negative controls, and R. Rycroft for correcting the English version of the manuscript.

Financial support: This work was supported by the Secretaria de Estado de Universidades e Investigacion through projects PB860546, SAL90-0960-CO2, and PB94-0865.

Authors' addresses: M. Jesus Aisa, Soledad Castillejo, Montserrat Gallego, Roser Fisa, M. Cristina Riera, Maria de Colmenares, Sara Torras, and Montserrat Portus, Laboratori de Parasitologia, Facultat de Farmacia, Universitat de Barcelona, Avda. Diagonal s/n, 08028 Barcelona, Spain. Xavier Roura, Hospital Clinic de Veterinaria, Facultat de Veterinaria, Universitat Autonoma de Barcelona, 08193 Bellaterra, Spain. Joan Sentis, Unitat de Bioestadistica, Departament de Salut Publica, Facultat de Medicina, Avda. Diagonal s/n, 08028 Barcelona, Spain.

Reprint requests: Montserrat Portus, Laboratori de Parasitologia, Facultat de Farmacia, Avda. Diagonal s/n, 08028 Barcelona, Spain.

\section{REFERENCES}

1. Abranches P, Lopes FJ, Silva FMC, Ribeiro MMS, Pires CA, 1983. Le Kala-azar au Portugal. III. Resultants d'une enquete sur la leishmaniose canine realisee dans les environs de Lisbonne. Comparaison des zones urbaines et rurales. Ann Parasitol Hum Comp 58: 307-315.

2. Acedo Sanchez C, Martin Sanchez J, Velez Bernal ID, Sanchis Marin MC, Louassini M, Maldonado JA, Morillas Marquez F, 1996. Leishmaniasis eco-epidemiology in the Alpujarra Region (Granada province, southern Spain). Int J Parasitol 25: 303-310.

3. Dye C, Killick-Kendrick R, Vitutia MM, Walton R, KillickKendrick M, Harith AE, Guy MW, Cañavate MC, Hasibeder G, 1992. Epidemiology of canine leishmaniasis: prevalence, incidence and basic reproduction number calculated from a cross-sectional serological survey on the island of Gozo, Malta. Parasitology 105: 35-41.
4. Portus M, Fisa R, Serra T, Gallego M, Mora R, 1987. Estudios seroepidemiologicos sobre la leishmaniosis canina en Cataluña. Med Vet 4: 569-575.

5. Fisa R, Portus M, Gallego M, Valls D, Aisa MJ, 1992. El diagnostico serologico de la leishmaniosis canina en la comarca del Priorat (Tarragona). Clin Vet Peq An 12: 231-236.

6. Mary C, Lamouroux D, Dunan S, Quilici M, 1992. Western blot analysis of antibodies to Leishmania infantum antigens: potential of the $14-\mathrm{kD}$ and $16-\mathrm{kD}$ antigens for diagnosis and epidemiologic purposes. Am J Trop Med Hyg 47: 764-771.

7. Rolland L, Zilberfarb V, Furtado A, Gentilini M, 1994. Identification of a 94-kilodalton antigen on Leishmania promastigote forms and its specific recognition in human and canine visceral leishmaniasis. Parasite Immunol 16: 599-608.

8. Cardeñosa N, Riera C, Cortes P, March F, Muñoz C, Portus M, Prats G, 1995. Detection and characterization by immunoblo analysis of potentially diagnostic Leishmania infantum polypeptides in human visceral leishmaniasis. Parasite Immunol 17: 509-516.

9. Abranches P, Santos-Gomes G, Rachamim N, Campino L, Schnur LF, Jaffe CL, 1991. An experimental model for canine visceral leishmaniasis. Parasite Immunol 13: 537-550.

10. Neogy AB, Vouldoukis I, Silva OA, Tselentis Y, Lascombe JC, Segalen T, Rzepka D, Monjour L, 1992. Serodiagnosis and screening of canine visceral leishmaniasis in an endemic area of Corsica: applicability of a direct agglutination test and immunoblot analysis. Am J Trop Med Hyg 47: 772-777.

11. Mancianti F, Falcone ML, Giannelli C, Poli A, 1995. Comparison between an enzyme-linked immunosorbent assay using a detergent-soluble Leishmania infantum antigen and indirect immunofluorescence for the diagnosis of canine leishmaniosis. Vet Parasitol 59: 13-21.

12. Carrera L, Fermin ML, Tesouro M, Garcia P, Rollan E, Gonzalez JL, Mendez S, Cuquerella M, Alunda JM, 1996. Antibody response in dogs experimentally infected with Leishmania infantum: infection course antigen markers. Exp Parasitol 82: 139-146.

13. Fisa R, Gallego M, Riera C, Aisa MJ, Valls D, Serra T, de Colmenares M, Castillejo S, Portus M, 1997. Serologic diagnosis of canine leishmaniasis by dot-ELISA. J Vet Diagn Invest 9: 50-55.

14. Suffia I, Quaranta JF, Eulalio MCM, Ferrua B, Marty P, Le Fichoux Y, Kubar J, 1995. Human T-cell activation by 14- and 18-kilodalton nuclear proteins of Leishmania infantum. Infect Immun 63: 3765-3771.

15. Louzir H, Tebourski F, Smith DF, Ben Ismail R, Dellagi K, 1994. Antibodies to Leishmania donovani infantum heat-shock protein 70 in human visceral leishmaniasis. J Infect Dis 169: $1183-1184$.

16. Quijada L, Requena JM, Soto M, Alonso C, 1996. During canine viscero-cutaneous leishmaniasis the anti-Hsp 70 antibodies are specifically elicited by the parasite protein. Parasitology 112: 277-284. 